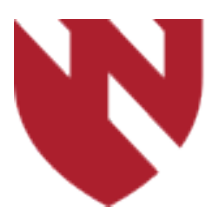

December 2019

\title{
Pharmacological Treatment of Status Epilepticus at UNMC: Assessment of Seizure Outcomes
}

\author{
Hae Young Baang \\ University of Nebraska Medical Center \\ Nicholas Swingle \\ University of Nebraska Medical Center \\ Kalyan Sajja \\ University of Nebraska Medical Center \\ Deepak Madhavan \\ University of Nebraska Medical Center \\ Olga Taraschenko \\ University of Nebraska Medical Center
}

Tell us how you used this information in this short survey.

Follow this and additional works at: https://digitalcommons.unmc.edu/gmerj

Part of the Higher Education Commons, and the Medicine and Health Sciences Commons

\section{Recommended Citation}

Baang, H., Swingle, N., Sajja, K., Madhavan, D., , Taraschenko, O. Pharmacological Treatment of Status Epilepticus at UNMC: Assessment of Seizure Outcomes. Graduate Medical Education Research Journal. 2019 Dec 13; 1(1).

https://digitalcommons.unmc.edu/gmerj/vol1/iss1/49

This Conference Proceeding is brought to you for free and open access by DigitalCommons@UNMC. It has been accepted for inclusion in Graduate Medical Education Research Journal by an authorized editor of DigitalCommons@UNMC.For more information, please contact digitalcommons@unmc.edu. 


\section{Pharmacological Treatment of Status Epilepticus at UNMC: Assessment of Seizure Outcomes}

\section{Creative Commons License}

\section{c) (1)@ $\Theta$}

This work is licensed under a Creative Commons Attribution-Noncommercial-No Derivative Works 4.0 License. 
$>0.05$, ANOVA). Postmortem evaluation revealed that 2-3 hepatic veins were transected by injury type $1,1-2$ portal vein branches plus 1 hepatic vein were transected by injury type 3 (LLL transection).

Conclusions: The hemorrhage severity of the central stellate injury was inadequate, while hemorrhage from excision of a proximal branch of the portal vein appeared to be too severe (i.e., the subjects died too quickly).
Near-transection of the hepatic LLL at its base appeared to yield hemorrhage of appropriate severity, as indicated by the $40 \% 1$-hour mortality and intermediate values for MAP, blood loss, and other variables. Our plan is to use this latter injury model in the development of therapies for noncompressible truncal hemorrhage.

https://doi.org/10.32873/unmc.dc.gmerj.1.1.047

\section{Exogenous Testosterone Supplementation as Cause of Ischemic Stroke, Mediated through Secondary Erythrocytosis \\ Fuad-al Ali, Amy Hellman, Pierre Fayad}

Mentor: Pierre Fayad

Program: Neurology

Background: The use of exogenous testosterone has become more prevalent in recent years but the effects of such use in regards to potential cerebrovascular disease have yet to come to a conclusion. In this case report, we discuss a patient who suffered an ischemic stroke due to testosterone supplementation.

Case presentation: A middle-aged patient with history of hypertension and hyperlipidemia that presented with falls, difficulty performing simple tasks and slurred speech. Patient had been taking testosterone injections every 6 months for the prior 2 years for loss of energy and reported low levels. Lab work revealed a hemoglobin of $20 \mathrm{~g} / \mathrm{dL}$ and a hematocrit of $51.5 \%$. MRI of the brain showed a left striatocapsular ischemic infarct. He was given the diagnosis of ischemic stroke due to polycythemia and thrombus formation secondary to testosterone supplementation.

Discussion: Testosterone and dihydrotestosterone (DHT) has both protective and adverse effects. Both low and high levels of testosterone and DHT have an association with ischemic stroke and there may be an optimal level that is safe.

Conclusion: The risk of ischemic stroke may be related to testosterone and DHT levels but no clear guidelines exist on optimal levels in supplementation. Thus, clinicians should be aware of the potential risk and monitor androgen and hematocrit levels.

https://doi.org/10.32873/unmc.dc.gmerj.1.1.048

\section{Pharmacological Treatment of Status Epilepticus at UNMC: Assessment of Seizure Outcomes} Hae Young Baang, Nicholas Swingle, Kalyan Sajja, Deepak Madhavan, Olga Taraschenko

Mentor: Olga Taraschenko

Program: Neurology

Objective: To assess the effectiveness of treatment for status epilepticus (SE) at UNMC and determine patient health outcomes related to the delayed control of seizures

Design/Methods: Retrospective chart review was performed on 1565 adult patients who underwent continuous video electroencephalography (cvEEG) for suspected seizures between January 1, 2012 and February 28, 2018.

Results: 49 patients had and were treated for 64 incidences of convulsive or nonconvulsive
SE recorded on cvEEG or clinical seizures longer than 5 min which were documented by a neurologist. Among 64 cases, 55 incidences required the second AED treatment, and 40 out of 55 cases were treated with the third AED subsequently. The mean seizure detection-to-needle time for the first, second, and third antiepileptic drugs were 167, 239, and 297 minutes, respectively. Benzodiazepines were administered as the first AED in only $46 \%$ of all treated cases. The duration of the hospital stays in patients treated with the first AED within 60 min after seizure detection was shorter compared to those whose treatment was delayed (23.0 vs.
33.6 days, $p=0.41$ ). The mortality rate in the former group was lower than that in the group which did not receive AEDs within $60 \mathrm{~min}$ (28.6 vs. $53.6 \%, \mathrm{p}=0.02$ ).

Conclusion: The current treatment approaches for SE at UNMC are inefficient with significant delay in delivery of medications after seizure detection; they may contribute to the prolonged hospital stay and increased mortality. In order to improve patient care, the existing protocol for the treatment of SE at UNMC must be revised.

https://doi.org/10.32873/unmc.dc.gmerj.1.1.049

\section{A Clinical and Cost Effectiveness Comparison of Traditional VNS Therapy to Aspire Sr (106)}

Krishna Mourya Galla, Kalyan Sajja, Christopher Wichman, Hongmei Wang, Deepak Madhavan

Mentor: Deepak Madhavan

Program: Neurology

Background: VNS Therapy has demonstrated overall cost savings in patients with drug- resistant epilepsy. The overall goal of this project is to evaluate the clinical and cost effectiveness of the Aspire SR 106 VNS (Vagus Nerve Stimulator) system, using retrospective patient data. We anticipate the extra dimension of closed-loop autostimulation in the Aspire 106 model will demonstrate more efficient utilization of health care resources. 